\title{
GOCE SGG and SST quick-look gravity field analysis
}

\author{
R. Pail ${ }^{1}$ and M. Wermuth ${ }^{2}$ \\ ${ }^{1}$ Institute of Geodesy, Graz University of Technology, Steyrergasse 30, A-8010 Graz, Austria \\ ${ }^{2}$ Institute of Astronomical and Physical Geodesy, Technical University Munich, Arcisstraße 21, D-80333 Munich, Germany
}

\begin{abstract}
The purpose of quick-look analysis in the framework of the GOCE data processing architecture is to analyse partial data sets of satellite gravity gradiometry (SGG) and satellite-to-satellite tracking in high-low mode (hl-SST), in order to derive a diagnosis of the system performance. The semi-analytic (SA) method for gravity field recovery from GOCE observations, which is based on Fast Fourier Transform, is used. From a theoretical point of view, a number of requirements (circular, exact repeat orbit, uninterrupted measurement time series) have to be fulfilled. However, in the present paper it is demonstrated, that the SA approach can also be applied in the practical case of non-circular, nonrepeat orbits, and data gaps in the GOCE measurement time series. The performance of this approach is assessed in 5 case studies on the basis of a realistic closed-loop simulation.
\end{abstract}

Key words. GOCE - semi-analytic approach - colored noise - data gaps - repeat orbit

\section{Introduction}

The satellite mission GOCE (Gravity field and steady-state Ocean Circulation Explorer; ESA, 1999), the first Core Mission of ESA's Earth Explorer Program, strives for a highaccuracy, high-resolution model of the Earth's static gravity field, represented by spherical harmonic coefficients complete up to degree and order 300 , which corresponds to a shortest spatial half-wavelength of less than $70 \mathrm{~km}$.

The GOCE measurement system is based on a sensor fusion concept: satellite-to-satellite tracking in the high-low mode (hl-SST) using GPS, plus on-board satellite gravity gradiometry (SGG). This data contains abundant information about the gravity field of the Earth on a near-global scale (except for the polar gaps due to the sun-synchronous orbit configuration), from very low (derived mostly from hl-SST) to high (derived mostly from SGG) frequencies.

Correspondence to: R. Pail (pail@geomatics.tu-graz.ac.at)
An adequate quality assessment of the observation time series is a crucial component for reaching the mission goals. The purpose of quick-look analysis is to analyse partial data sets of SGG and hl-SST data, in order to derive a diagnosis of the GOCE system performance. The quick-look analysis will be performed in parallel to the mission, and successive preliminary gravity field models are computed in short time intervals based on the partial SST and SGG data. The major goal is to detect potential distortions of statistical significance (e.g. systematic errors), and to give a feedback to mission control with a minimum latency after the actual measurements.

This can be achieved by several strategies. Either the successively produced gravity field solutions are compared with an a priori defined reference model, where in a statistical test procedure the corresponding variance/covariance information is included. Another strategy is the spectral analysis of the residuals of the adjustment (Pail, 2002) and the comparison with the a priori known error power spectral density (PSD) of the gradiometer, again based on standard statistical tests, and the estimation of variance components.

The quick-look analysis tool is based on the semi-analytic approach, a fast algorithm for gravity field recovery from SGG and SST observations, using the sparseness of the normal equation matrix and a number of additional simplifying assumptions (e.g. circular orbit, exact repeat orbit, uninterrupted measurement time series). In the present paper we concentrate on strategies to overcome these restrictions in practical application. Special concern is given to strategies to cope with the colored noise behaviour of the gradiometer.

The performance of the quick-look tool is assessed on the basis of a realistic GOCE mission simulation, regarding the accuracy of the results, and special concern is given to the minimization of the computation effort. The numerical simulations include a realistic colored noise model and the problem of partial data sets and data gaps. 


\section{Functional model}

While in the direct gravity field solution strategies the observations are regarded as functions of the geographical location, they can also be considered as a periodic time series for one repeat period (cf. Rummel et al., 1993; Sneeuw, 2000). Assuming a circular orbit, the gravitational potential $V$ and also second order derivatives $V_{\alpha \beta}$ can be rewritten as a Fourier series

$$
V_{\alpha \beta}(t)=\sum_{m} \sum_{k}\left[A_{k m} \cos \psi_{k m}(t)+B_{k m} \sin \psi_{k m}(t)\right]
$$

where $\psi_{k m}(t)$ is related to the two fundamental frequencies $\omega_{o}$ (satellite orbit revolution) and $\omega_{e}$ (Earth's rotation).

The spherical harmonic coefficients $\bar{C}_{l m}, \bar{S}_{l m}$ of the same order $m$ are lumped together in a linear way to compose the Fourier coefficients $A_{k m}$ and $B_{k m}$ :

$$
\begin{aligned}
& A_{k m}= \\
& \frac{G M}{R} \sum_{l=l_{\min }[2]}^{l_{\max }}\left(\frac{R}{r}\right)^{l+1} \lambda_{l k}^{(\alpha \beta)} \bar{F}_{l m}^{k}(I)\left\{\begin{array}{c}
\bar{C}_{l m} \\
-\bar{S}_{l m}
\end{array}\right\}_{l-m}^{l-m} \text { odd } \\
& B_{k m}= \\
& \frac{G M}{R} \sum_{l=l_{\min }[2]}^{l_{\max }}\left(\frac{R}{r}\right)^{l+1} \lambda_{l k}^{(\alpha \beta)} \bar{F}_{l m}^{k}(I)\left\{\begin{array}{c}
\bar{S}_{l m} \\
\bar{C}_{l m}
\end{array}\right\}_{l-m}^{l-m} \text { oden }
\end{aligned}
$$

where $\bar{F}_{l m}^{k}(I)$ denotes the inclination function, which depends on the orbit inclination $I$. The spectral transfer coefficients $\lambda_{l k}^{(\alpha \beta)}$ express the relation between the spherical harmonics $\bar{C}_{l m}, \bar{S}_{l m}$ and a specific observable $V_{\alpha \beta}$.

In this SA approach (also frequently denoted as timewise approach in the frequency domain) in the first step the Fourier coefficients ('lumped coefficients') $A_{k m}, B_{k m}$ are computed by FFT techniques, and in the second step the harmonic coefficients $\bar{C}_{l m}, \bar{S}_{l m}$ are adjusted order by order from the pseudo-observations $A_{k m}, B_{k m}$, assuming a blockdiagonal structure of the normal equation system. In a strict sense, this algorithm only holds for

- an uninterrupted sequence of observations

- with constant sampling rate, measured

- along a circular,

- exact repeat orbit.

- The number of nodal days $N_{E}$ and orbital revolutions per repeat cycle $N_{0}$ must not have a common divisor, and

- for the maximum resolvable harmonic degree holds: $l_{\max }<N_{0} / 2$.

Deviations from these requirements, such as non-circular orbits, non-repeat orbits, or data gaps in the measurement time series, are incorporated by means of an iterative procedure. Since this approach partially works in frequency domain, it provides a direct access to the spectral instrument characteristics, which can be implemented in the processing stream in a very direct and easy way. The spectral gradiometer properties can be described by the frequencydependent error variances $\sigma_{k m}$, which are included as a diagonal variance-covariance matrix of the pseudo-observations $A_{k m}, B_{k m}$ in the course of the adjustment of the harmonic coefficients.

\section{Numerical simulations and results}

The numerical test simulation is based on the following configuration:

- Orbit: non-circular, sun-synchronous 61 days/982 revolutions repeat orbit with a mean altitude of $250 \mathrm{~km}$.

- Measurement time series (either $V_{z z}$ or all 3 main diagonal elements $V_{x x}, V_{y y}, V_{z z}$ of the gradiometer tensor) with a sampling interval of $10 \mathrm{~s}$.

- Signals based on the Earth model OSU91a (Rapp et al., 1991) complete up to degree and order 72.

- Realistic colored noise according to the Alenia (2001) specifications, generated by an auto-regressive moving average (ARMA) process (cf. Schuh et al., 1996; Pail and Plank, 2002).

For the sake of consistency a test data set containing signals complete up to degree and order 72 is used throughout the paper, because the major intention was to demonstrate the effect of different configurations on the gravity solution. However, it should be emphasized, that the quick-look software was already successfully applied to systems up to degree and order 250 .

\subsection{Case Study 1: Radial component $V_{z z}-$ no noise}

In the first case study, the method was first applied to "perfect measurements". The ground-track of the orbit closes exactly, and the simulated gradiometer measurements of the $V_{z z}$ component contain no noise. Figure 1 shows the convergence behaviour of the SA method in terms of the deviations of the estimated coefficients from the initial 'true' OSU91a model represented by a degree rms

$\sigma_{l}^{(i)}=$

$\sqrt{\frac{1}{2 l+1} \sum_{m=0}^{l}\left[\left(\bar{C}_{l m, i}^{(e s t)}-\bar{C}_{l m}^{(O S U)}\right)^{2}+\left(\bar{S}_{l m, i}^{(e s t)}-\bar{S}_{l m}^{(O S U)}\right)^{2}\right]}$

for the first $i=1, \ldots 17$ iterations. Here (est) denotes the adjusted quantities and $(O S U)$ refers to our reference model OSU91a. Figure 1 demonstrates, that the computation converges to the level of the numerical accuracy, and the final 


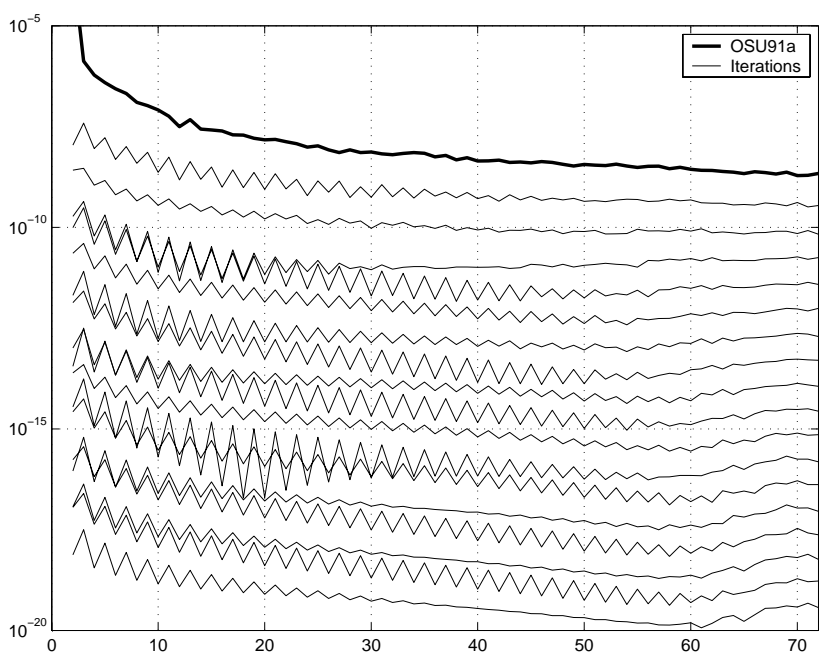

Fig. 1. Proof of concept: $V_{z z}$ signals, no noise superimposed.

solution perfectly reproduces the input gravity field. This result is both a proof of the concept and of the correct implementation of the algorithm.

In the following case studies the behaviour of the method in the case of more realistic measurements will be demonstrated.

\subsection{Case Study 2: Colored noise}

According to the Alenia (2001) specifications, the gradiometer has a colored noise characteristics. The total error spectral density $S(f)$ of the gravity gradient tensor diagonal elements $V_{x x}, V_{y y}, V_{z z}$ is flat and shall not exceed $S(f) \leq 4 m E / \sqrt{H z}$ in the measurement bandwidth $(5 \mathrm{mHz} \leq f \leq 100 \mathrm{~m} \mathrm{~Hz})$ in the local orbital reference frame. Outside the measurement bandwidth, the upper limit of the total error spectrum is specified by $S(f) \leq 4 \frac{f_{\min }}{f} \mathrm{mE} / \sqrt{\mathrm{Hz}}$ below $f_{\min }=5 \mathrm{mHz}$ and $S(f) \leq 4\left(\frac{f}{f_{\max }}\right)^{2} \mathrm{mE} / \sqrt{\mathrm{Hz}}$ above $f_{\max }=100 \mathrm{mHz}$, forming a spectrum of trapezoidal shape in log-log scale. Figure 2 shows a realization of the noise spectrum for the component $V_{z z}$, generated by an ARMA process, which will be superimposed to the measurement time series in the following simulations.

Figure 3 displays the convergence behaviour in the case of colored noise, where the solid curve indicates the final solution. Using not only the $V_{z z}$ component, but all three main diagonal elements of the gradiometer tensor $V_{x x}, V_{y y}$ and $V_{z z}$ (including corresponding colored noise time series), leads to slightly improved results (cf. Fig. 3, light curve). The final result is obtained in less than $30 \mathrm{~min}$ on a standard PC.

Figure 4 illustrates the accuracy of the coefficient estimates in terms of gravity anomaly deviations from the initial OSU91a model. Figure 4a refers to the $V_{z z}$ solution, whereas Fig. $4 \mathrm{~b}$ shows the deviations applying all three tensor components $V_{x x}, V_{y y}, V_{z z}$. The dominant long-wavelength error

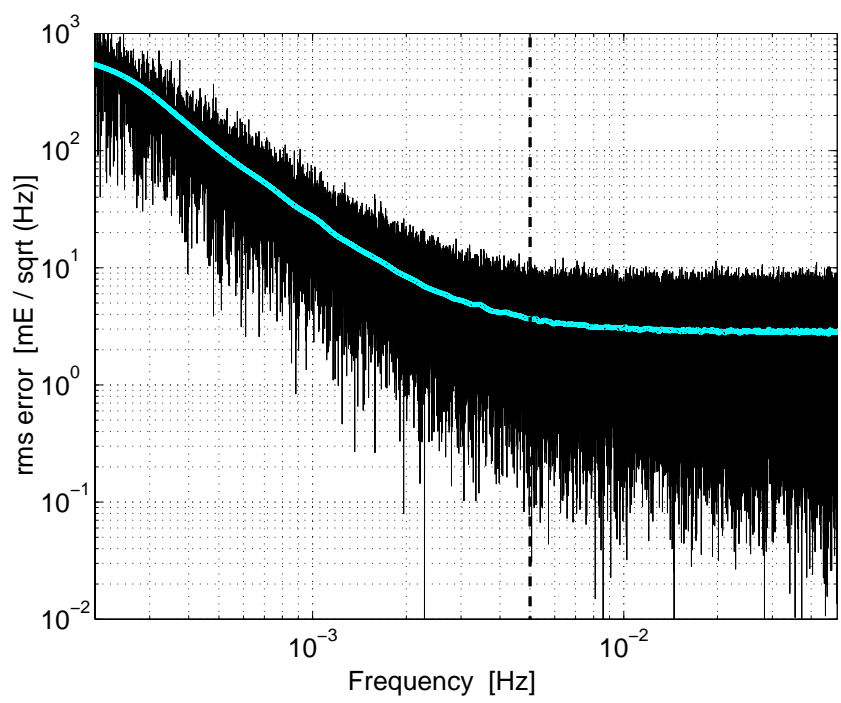

Fig. 2. Noise PSD for $V_{z z}$ component.

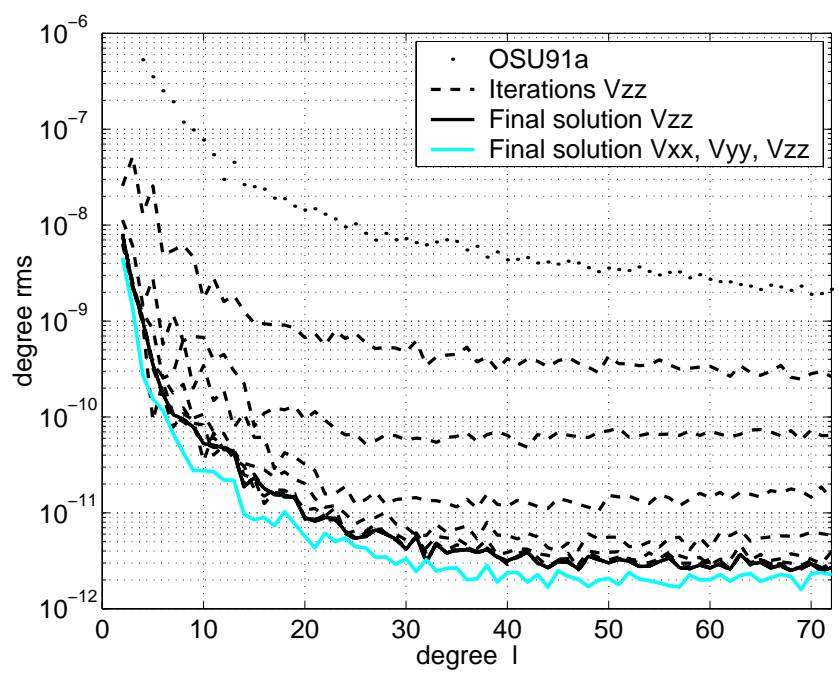

Fig. 3. Convergence behavior of the SA approach: colored noise superimposed to measurement time series.

behaviour reflects the decreased measurement accuracy below the measurement bandwidth (cf. Fig. 2). In practice, this problem will largely be captured by the SST component. The solution in Fig. $4 \mathrm{~b}$ is mainly stabilized by the additional $V_{y y}$ component.

\subsection{Case Study 3: Large data gaps + non-repeat orbit}

In the previous case studies, a nearly perfect repeat orbit and a continuous data stream were assumed. If the orbit groundtrack does not close exactly, or if the measurement time series contain data gaps, the requirement of periodicity for the FFT is not fulfilled. Both effects lead to a distortion of the Fourier spectrum, and thus of the lumped coefficients. How- 


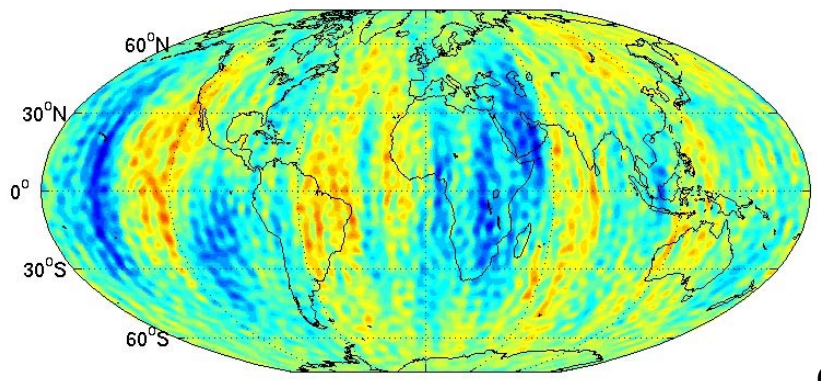

(a)

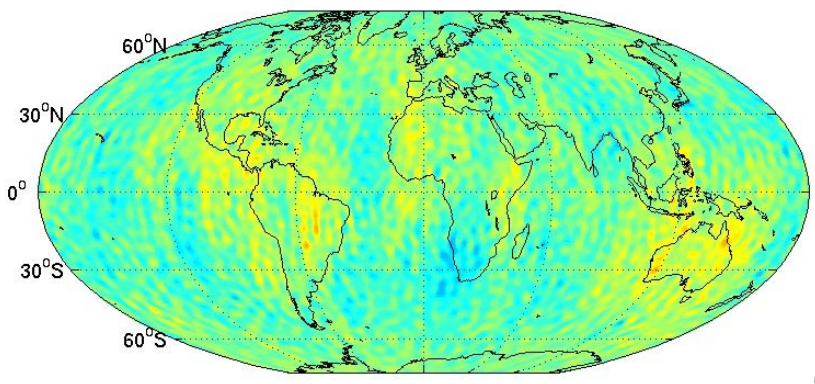

(b)

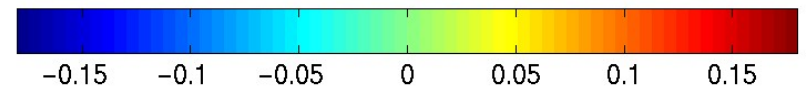

Fig. 4. Gravity anomaly deviations $\Delta g$ [mGal] $(1 \mathrm{mGal}=$ $10^{-5} \mathrm{~m} \mathrm{~s}^{-2}$ ) from the 'true' OSU91A reference model applying (a) $V_{z z}$ only; (b) all 3 main diagonal tensor components $V_{x x}, V_{y y}$, $V_{z z}$.

ever, as it will be demonstrated in the present case study, small lateral offsets (below $100 \mathrm{~km}$ ), and data gaps of up to $50 \%$ of the whole repeat cycle, do not pose a problem for the iterative solution (provided that the number of data is sufficient for the selected maximum degree of resolution to ensure redundancy and a global data distribution), but reduce the rate of convergence. A series of tests with varying lateral offsets and increasing data gaps were carried out in a noisefree scenario. Figure 5 illustrates, that the convergence rate (the ratio between the residuals after and before one iteration step) depends on the lateral offset, and on the size of the data gaps.

\subsection{Case Study 4: Large data gaps + colored noise}

In this simulation we investigate the effect of data gaps in the case of a realistic colored noise applied to the measurement time series. Here the last $30 \%$ of the $V_{x x}, V_{y y}$ and $V_{z z}$ data sets are eliminated. Figure 6 illustrates the convergence behavior of this configuration, where the black solid curve indicates the final solution. As a reference, the result using the complete data sets is plotted in light color. Compared with the uninterrupted case (cf. Sect. 3.2), the data gap leads to a slower rate of convergence, but the accuracy of the results is only slightly decreased, which is simply due to the fact that a smaller number of data was processed. The speed of convergence can be improved using a priori gravity field information for filling the data gaps in the first iteration.
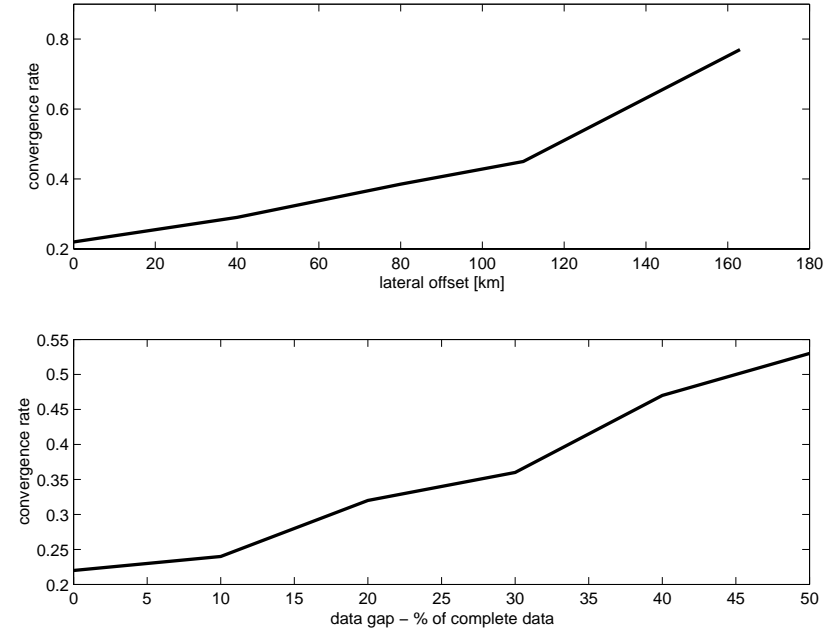

Fig. 5. Convergence rates in dependence of (a) the lateral offset of a non-closing orbit, and (b) the size of a data gap.

\subsection{Case Study 5: Short-term data gaps + colored noise}

In practice, in addition to large data gaps and partial data sets also short-term failure of the gradiometer has to be expected, leading to short data gaps in the GOCE measurement time series. In this last case study, we simulate short-term gaps (10 s to $10 \mathrm{~min}$ ), which occur randomly about once per orbit revolution.

Figure 7 shows the convergence behaviour of this configuration. The convergence rate can be increased by filling the data gaps with a priori gravity field information - in the present case simulated gravity gradients based on the Earth model EGM96 (Lemoine et al., 1998) are used - in the first iteration, and by interpolation within the data gaps in the subsequent iterations. Obviously, the procedure converges very fast towards an optimum solution. For comparison, the final solution of the uninterrupted case (cf. Sect. 3.2) is displayed in light color, demonstrating, that there is no significant difference between the two solutions.

\section{Conclusions}

In this paper we present the semi-analytic approach as an extremely fast method which suggests itself to be used as a quick-look tool, applied to derive a diagnosis of the GOCE system performance. It is demonstrated that the theoretical restrictions of the method (uninterrupted measurement time series, circular, exact repeat orbit, ...) can be overcome in practical application by an iterative procedure.

The method was successfully applied in the case of realistic orbits and colored noise characteristics of the gradiometer. It can be used to analyse partial data sets, and it works also in the case of large or short-term data gaps in the GOCE measurement time series. 


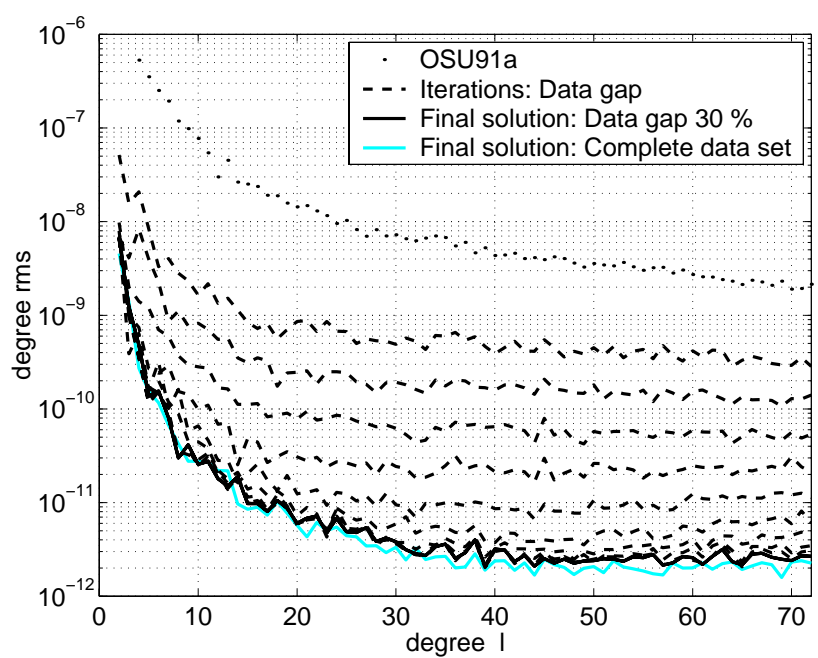

Fig. 6. Convergence behavior of the SA approach in the case of a data gap of $30 \%$.

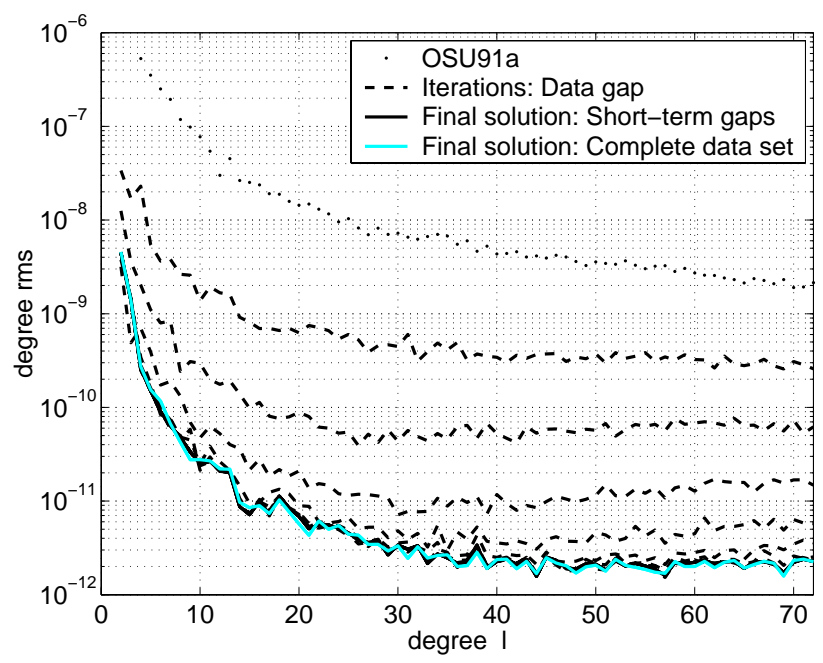

Fig. 7. Convergence behavior of the SA approach in the case of many short-term data gaps.

\section{References}

Alenia: Performance requirements and budgets for the gradiometric mission. Technical Note, GOC-TN-AI-0027, Alenia Spazio, Turin, Italy, 2001.

ESA: Gravity Field and Steady-State Ocean Circulation Mission, Reports for mission selection, The four candidate Earth explorer core missions, SP-1233(1), European Space Agency, Noordwijk, 1999.

Lemoine, F. G., Kenyon, S. C., Factor, J. K., Trimmer, R. G., Pavlis, N. K., Chinn, D. S., Cox, C. M., Klosko, S. M., Luthcke, S. B., Torrence, M. H., Wang, Y. M., Williamson, R. G., Pavlis, E. C., Rapp, R. H., and Olson, T. R.: The Development of the Joint NASE GSFC and the National Imagery and Mapping Agency (NIMA) Geopotential Model EGM96, National Aeronautics and Space Administration, Goddard Space Flight Center, Greenbelt, Maryland, 1998.

Pail, R.: In-orbit calibration and local gravity field continuation problem. ESA-Project, "From Eötvös to mGal+", ESA/ESTEC Contract 14287/00/NL/DC, Final Report, WP 1, 9-112, European Space Agency, Noordwijk, (www-geomatics.tu-graz.ac.at/ mggi/research/e2mgp/e2mgp.htm), 2002.

Pail, R. and Plank, G.: Assessment of three numerical solution strategies for gravity field recovery from GOCE satellite gravity gradiometry implemented on a parallel platform, J. Geod., 76, 462-474, 2002.

Rapp, R., Wang, Y., and Pavlis, N.: The Ohio state 1991 geopotential and sea surface topography harmonic coefficient models, OSU Report 410, Department of Geodetic Science and Surveying, The Ohio State University, Columbus, 1991.

Rummel, R., van Gelderen, M., Koop, R., Schrama, E., Sansó, F., Brovelli, M., Miggliaccio, F., and Sacerdote, F.: Spherical harmonic analysis of satellite gradiometry, Neth. Geod. Comm., Publications on Geodesy, no. 39, Delft, The Netherlands, 1993.

Schuh, W.-D., Sünkel, H., Hausleitner, W., and Höck, E.: Refinement of Iterative Procedures for the Reduction of Spaceborne Gravimetry Data, ESA project CIGAR IV, Final report. ESA contract 152163-ESA study ESTEC/JP/95-4-137/MS/nr, European Space Agency, Noordwijk, 157-212, 1996.

Sneeuw, N.: A semi-analytical approach to gravity field analysis from satellite observations, Dissertation, DGK, Reihe C, Munich, no. 527, Bayerische Akademie d. Wissenschaften, Munich, 2000 . 